\title{
Inhibition of phosphodiesterase 4D decreases the malignant properties of DLD-1 colorectal cancer cells by repressing the AKT/mTOR/Myc signaling pathway
}

\author{
DONG UK KIM ${ }^{1 *}$, JEHYUN NAM ${ }^{1 *}$, MATTHEW D. CHA ${ }^{2}$ and SANG-WOO KIM ${ }^{1}$ \\ ${ }^{1}$ Department of Biological Sciences, Pusan National University, Busan 46241, Republic of Korea; \\ ${ }^{2}$ Department of Biological Sciences, The University of Texas at Dallas, Richardson, TX 75080, USA
}

Received May 14, 2018; Accepted December 3, 2018

DOI: $10.3892 / 01.2019 .9996$

\begin{abstract}
Colorectal cancer (CRC) is a complex disease involving numerous genetic abnormalities. One of the major characteristics of CRC is enhanced Wnt signaling caused by loss-of-function mutations in the adenomatous polyposis coli (APC) gene. Previously, it has been demonstrated that the majority of malignant phenotypes following APC deletion in adult murine small intestines could be rescued when Myc, a downstream target of the Wnt pathway, was deleted. This indicated that Myc is a critical regulator of CRC development following APC loss. Previous studies reported that cyclic adenosine 3',5'-monophosphate (cAMP) can influence the AKT/mammalian target of rapamycin (mTOR) survival pathway in cancer and Myc is a critical downstream molecule of AKT/mTOR signaling. Phosphodiesterase 4D (PDE4D), a member of the cAMP-specific PDE4 family, has been associated with drug resistance in CRC. However, the association between PDE4D and Myc remains unclear. To investigate the potential role of PDE4D in Myc regulation in CRC, the present study evaluated the expression levels of PDE4 subtypes in DLD-1 CRC cells. Additionally, the effects of PDE4 inhibitors on Myc expression and oncogenic properties were analyzed by western blot analysis, reverse transcription-quantitative polymerase chain reaction, colony formation and soft agar assays. It was demonstrated that cAMP/PDE4D signals serve a critical role in regulating Myc expression in DLD-1 CRC cells. Furthermore, PDE4D was identified to be a main hydrolyzer of cAMP and suppression of PDE4D using selective inhibitors of PDE4
\end{abstract}

Correspondence to: Professor Sang-Woo Kim, Department of Biological Sciences, Pusan National University, 63 Beon-gil 2 Busandaehag-ro, Busan 46241, Republic of Korea

E-mail: kimsw@pusan.ac.kr

${ }^{*}$ Contributed equally

Key words: diseases, colorectal cancer, drug effects, oncogene, tumor suppressor, cyclic adenosine 3',5'-monophosphate, phosphodiesterase 4D, Myc increased intracellular cAMP levels, which resulted in a marked decrease in the oncogenic properties of DLD-1 cells, including colony formation, cell proliferation and anchorage-independent growth. Notably, the current data imply that cAMP represses Myc expression via the downregulation of AKT/mTOR signaling, which was abolished by high PDE4D activities in DLD-1 cells. Additionally, a natural polyphenol resveratrol in combination with forskolin elevated the concentration of cAMP and enhanced the expression of Myc and the malignant phenotype of DLD-1 cells, reproducing the effect of known chemical inhibitors of PDE4. In conclusion, the present study identified that $\mathrm{cAMP} / \mathrm{PDE} 4 \mathrm{D}$ signaling is a critical regulator of Myc expression in DLD-1 and possibly other CRC cells.

\section{Introduction}

Despite recent developments of various screening methods and procedures for early detection of cancer, colorectal cancer (CRC) remains one of the most fatal cancer types globally, with almost 700,000 mortalities reported in 2012 (1) The overall incidence rate of CRC is decreasing; however, the mortality rate for adults $<50$ years old in USA is increasing, which makes it essential to improve understanding of CRC at the molecular and cellular level (2). A total of three major pathways are involved in the progression of CRC, including the cytosine-phosphate-guanine island methylator phenotype (CIMP) pathway, the microsatellite instability (MSI) pathway and the chromosomal instability (CIN) pathway (3). The CIMP pathway is associated with the hypermethylation of gene promoters. In subsets of CIMP-positive tumors, mutations in BRAF are present (4). BRAF that is activated by mutations is strongly associated with the CIMP pathway and this association has been reported in CRC cases (4). The MSI pathway results from impaired DNA mismatch repair (MMR), which causes mutations to accumulate (3). Inactivation of MMR induces the loss of the adenomatous polyposis coli (APC) gene and mutates cell differentiation genes (5). One of the early events of the CIN pathway is the loss of APC (5). APC-mutant cells slowly accumulate to produce a polyp, followed by the inactivation of additional tumor suppressor genes and the activation of proto-oncogenes, including c-Src, Myc and KRAS (5). Mutations in the small GTPase KRAS induce large adenomas 
and early carcinomas (6). Subsequently, the loss of SMAD4 and tumor protein 53 has been identified in carcinomas (6).

Genes encoding signaling molecules in the Wnt/enes encoding signhave been revealed to be frequently mutated, resulting in abnormal activation of this signaling cascade. For example, familial adenomatous polyposis, an autosomal dominant syndrome, is largely associated with germline mutations in the APC tumor suppressor gene, which is a negative regulator of the Wnt pathway (7). Inactivating mutations within APC have also been identified in $>80 \%$ of sporadic CRC cases and $50 \%$ of patients with CRC with wild-type APC exhibit activating mutations within the pathwayrgely al which demonstrates a clear association between colorectal neoplasia and aberrant Wnt signals (8). These data indicate that the Wnt pathway is a promising therapeutic target for the treatment of CRC. The Wnt/promisin pathway regulates numerous target genes, including Myc, cyclin D1 and vascular endothelial growth factor (9-11). However, it is noteworthy that the malignant phenotype induced by APC deficiency was rescued upon Myc deletion in murine intestine, implying that aberrant Wnt signaling exerts its effect predominantly through the upregulation of Myc expression (12). Therefore, strategies have been devised to therapeutically target Myc (13). For example, inhibitors of proviral integration site for Moloney murine leukemia virus (PIM) kinases, including PIMi and SMI-4a, have been developed to decrease Myc protein stability (13). Additionally, 10058-F4 is a chemical drug that inhibits Myc-Max dimerization and their binding to DNA (13). A better understanding of the mechanism underlying Myc deregulation may result in more efficient targeting of Myc in Myc-dependent CRC.

Cyclic adenosine 3',5'-monophosphate (cAMP), an intracellular second messenger, was first identified by Sutherland and Rall in $1958(14,15)$. Since then, its broad impact on cellular physiology, including its role in inflammation, cell cycle progression and cytotoxicity has been well characterized (16-18). Adenylyl cyclase and phosphodiesterases (PDEs) generate and hydrolyze cAMP (18). Previous studies using various types of cancer models have indicated that cancer cells, including B lymphoma cells and CRC cells, exhibit low levels of intracellular cAMP; conversely, the elevation of cAMP levels by inhibition of PDE4 activities can result in growth arrest and/or cell death (19-22). These data indicate that PDE4 serves a critical role in the regulation of cAMP levels in these cells. PDE4 consists of four different isoforms, PDE4A, 4B, $4 \mathrm{C}$ and $4 \mathrm{D}$, and a previous study reported that PDE4D is a target of microRNA (miR)-139, whose expression is in turn regulated by the p53 tumor suppressor (23). The expression of miR-139 is inversely correlated with that of PDE4D in CRC samples (23), which indicates that PDE4D is a potential oncogene. Previous studies demonstrated that aberrant Myc expression is causally associated with the pathophysiology of CRC (5); however, how Myc is regulated by cAMP/PDE4D signaling remains unknown. The present study aimed to elucidate the mechanism underlying Myc deregulation, which may result in more effective targeting of Myc in CRC.

\section{Materials and methods}

Cell culture and reagents. Human DLD-1 CRC cells (Korean Cell Line Bank; Korean Cell Line Research Foundation, Seoul,
Korea) were maintained at $37^{\circ} \mathrm{C}$ in $5 \% \mathrm{CO}_{2}$ in RPMI-1640 medium (Gibco; Thermo Fisher Scientific, Inc., Waltham, MA, USA) supplemented with $10 \%$ fetal bovine serum (Capricorn Scientific GmbH, Ebsdorfergrund, Germany).

The following antibodies were used in the present study: Anti-phosphorylated (p)AKT (1:2,000 dilution; catalog no. 9271; Cell Signaling Technology, Inc., Danvers, MA, USA), anti-AKT (1:2,000 dilution; catalog no. 9272; Cell Signaling Technology, Inc.), anti-eukaryotic translation initiation factor 4E-binding protein 1 (1:2,000 dilution; 4EBP1; catalog no. 9452; Cell Signaling Technology,Inc.), anti-p4EBP1 (1:2,000 dilution; catalog no. 9459; Cell Signaling Technology, Inc.), anti-c-Myc (1:2,000 dilution; catalog no. ab32072; Abcam, Cambridge, UK), anti- $\beta$-actin (1:5,000 dilution; catalog no. sc-47778; Santa Cruz Biotechnology, Inc., Dallas, TX, USA), and horseradish peroxidase (HRP)-conjugated anti-rabbit (1:10,000 dilution; catalog no. A120-101p; Bethyl Laboratories, Inc., Montgomery, TX, USA) and anti-mouse (1:10,000 dilution; catalog no. A90-116p-33; Bethyl Laboratories, Inc.) antibodies.

The following chemicals were used: Dimethylsulfoxide (DMSO) (catalog no. D1370.0100; Duchefa Biochemie, Haarlem, Netherlands), Forskolin (catalog no. BML-CN100; Enzo Life Sciences, Inc., Farmingdale, NY, USA), rolipram (catalog no. R1012; AG Scientific, Inc., San Diego, CA, USA), roflumilast (catalog no. 2675-50; BioVision, Inc., Milpitas, CA, USA), rapamycin (catalog no. sc3504; Santa Cruz Biotechnology, Inc.), JQ1 (catalog no. A1910; ApexBio Technology, Houston, TX, USA) and resveratrol (catalog no. 554325; Merck KGaA, Darmstadt, Germany).

cAMP assays. DLD-1 cells ( $3 \times 10^{5}$ cells/well) were seeded in 6 -well plates. Following incubation overnight at $37^{\circ} \mathrm{C}$ in a $5 \%$ $\mathrm{CO}_{2}$ incubator, the cells were treated with forskolin (20 or $40 \mu \mathrm{M}$ ) for $2 \mathrm{~h}$ at $37^{\circ} \mathrm{C}$, following pre-incubation with rolipram $(40 \mu \mathrm{M})$ for $6 \mathrm{~h}$ or resveratrol $(20 \mu \mathrm{M})$ for $16 \mathrm{~h}$ at $37^{\circ} \mathrm{C}$. The concentration of cAMP was determined using a Parameter cAMP assay kit (catalog no. KGE002B; R\&D Systems, Inc., Minneapolis, MN, USA), according to the manufacturer's protocol.

Western blot analysis. DLD-1 ( $3 \times 10^{5}$ cells/well) were seeded in 6-well dishes and treated with DMSO, forskolin (10 or $20 \mu \mathrm{M}$ for $16 \mathrm{~h}$ ), rolipram (20 or $40 \mu \mathrm{M}$ for $16 \mathrm{~h}$ ), roflumilast ( $40 \mu \mathrm{M}$ for $16 \mathrm{~h}$ ), JQ1 ( 0.5 or $1 \mu \mathrm{M}$ for $24 \mathrm{~h}$ ), rapamycin ( 25 or $50 \mathrm{nM}$ for $24 \mathrm{~h}$ ), and resveratrol $(20 \mu \mathrm{M}$ for $\sim 48-72 \mathrm{~h})$ at $37^{\circ} \mathrm{C}$. Following treatment, cells were harvested by scraping and rinsed with PBS. The cells were lysed in radioimmunoprecipitation assay buffer (Elpis Biotech, Inc., Daejeon, Korea) mixed with sodium vanadate $(1 \mathrm{mM}$; Sigma-Aldrich; Merck KGaA), $\beta$-glycerol phosphate (50 mM; Sigma-Aldrich; Merck $\mathrm{KGaA}$ ), protease inhibitor (1X; G-Biosciences, St. Louis, MO, USA), EDTA (5 mM; G-Biosciences) and $\beta$-mercaptoethanol (142 mM; Bioworld Technology, Inc., St. Louis Park, MN, USA). The lysates were separated on 10 or $15 \%$ polyacrylamide gels, transferred onto polyvinylidene difluoride membranes and then blocked with $1 \%$ bovine serum albumin for $1 \mathrm{~h}$ at room temperature (MP Biomedicals, LLC, Santa Ana, CA, USA) dissolved in TBS and Tween-20. The membranes were probed with the aforementioned primary antibodies overnight at $4^{\circ} \mathrm{C}$ and HRP-conjugated secondary antibodies for $1 \mathrm{~h}$ at 
room temperature. Subsequently, the signals were visualized using enhanced chemiluminescent reagent (EzWestLumi plus; ATTO Corporation, Tokyo, Japan) and analysis of the relevant protein levels was performed using Luminograph II image analysis software (WSE-6100; ATTO Corporation).

Clonogenic assay. To measure clonogenicity, $1 \times 10^{3}$ DLD-1 cells/well were seeded in 6-well plates, incubated overnight at $37^{\circ} \mathrm{C}$ and then treated with DMSO (control), $20 \mu \mathrm{M}$ forskolin and/or $40 \mu \mathrm{M}$ rolipram/roflumilast for 7 days at $37^{\circ} \mathrm{C}$. The visible colonies that developed were stained with $0.5 \%$ crystal violet solution in $25 \%$ methanol, and counted with the naked eye at room temperature.

Soft agar assay. DLD-1 cells $\left(5 \times 10^{3}\right.$ cells/well) were mixed with $0.2 \%$ top agar (catalog no. A9414-5G; Sigma-Aldrich; Merck KGaA) and seeded on $0.6 \%$ bottom agar in 12 -well plates to visualize their anchorage-independent growth. The treatment conditions (DMSO, $20 \mu \mathrm{M}$ forskolin and $40 \mu \mathrm{M}$ rolipram/roflumilast) were maintained for 21 days at $37^{\circ} \mathrm{C}$ with $5 \% \mathrm{CO}_{2}$. The colonies were then stained with $0.005 \%$ crystal violet solution for $1 \mathrm{~h}$ at room temperature. The colonies were counted using a light microscope (Olympus CKX41; Olympus Corporation, Tokyo, Japan) at x20 magnification.

Cell counting. DLD-1 cells were seeded on day 0 at a density of $1 \times 10^{5}$ cells/well into 6-well plates and cultured with DMSO (control), $20 \mu \mathrm{M}$ forskolin and $40 \mu \mathrm{M}$ rolipram/roflumilast for 6 days at $37^{\circ} \mathrm{C}$. The cells were detached by trypsinization at $37^{\circ} \mathrm{C}$ and washed with PBS for counting with a hemocytometer at x100 magnification every day for 6 days.

Reverse transcription-quantitative polymerase chain reaction $(R T-q P C R)$. To quantify mRNA levels of PDE4A, 4B, $4 \mathrm{C}$ and $4 \mathrm{D}, \mathrm{RT}-\mathrm{qPCR}$ was performed and calculated using the $2^{-\Delta \Delta \mathrm{Cq}}$ method (20). TATA-box binding protein (TBP) was used as an internal control. First, $1 \times 10^{6}$ DLD-1 cells/well were seeded in 6-well plates overnight at $37^{\circ} \mathrm{C}$ and subsequently treated with DMSO, $40 \mu \mathrm{M}$ forskolin for $2 \mathrm{~h}, 40 \mu \mathrm{M}$ rolipram for $6 \mathrm{~h}$, or a combination of forskolin and rolipram at $37^{\circ} \mathrm{C}$. Finally, DLD-1 cells were harvested by scraping. Total RNA from DLD-1 CRC cells ( $1 \times 10^{6}$ cells per sample) was extracted using Tri-RNA Reagent (Favorgen Biotech Cooperation, Kaohsiung, Taiwan). cDNA was synthesized using PrimeScript ${ }^{\mathrm{TM}}$ RT reagent kit with gDNA Erase (Takara Bio, Inc., Otsu, Japan), according to the manufacturer's protocol. qPCR was then performed using a CFX connect ${ }^{\mathrm{TM}}$ Realtime system (Bio-Rad Laboratories, Inc., Hercules, CA, USA) and TOPreal $^{\mathrm{TM}}$ qPCR 2X PreMIX (SYBR ${ }^{\circledR}$ Green with low ROX; Enzynomics, Inc., Daejeon, Korea), according to the manufacturer's protocols. The primer sequences for PDE4A, 4B and 4D were as described previously $(19,23,24)$. The primer sequences for PDE4C and TBP were as follows: PDE4C forward, 5'-AGTCCCATGTGTGACAAGCA-3', and reverse, 5'-TCTGGTTGTCGAGGGGTAAG-3'; and TBP forward, 5'-TATAATCCCAAGCGGTTTGCTGCG-3', and reverse, 5'-AATTGTTGGTGGGTGAGCACAAGG-3'. The following PCR amplification cycles were used: $95^{\circ} \mathrm{C}$ for $15 \mathrm{~min}$, and 40 cycles of $95^{\circ} \mathrm{C}$ for $15 \mathrm{sec}, 59^{\circ} \mathrm{C}$ for $15 \mathrm{sec}$ and $27^{\circ} \mathrm{C}$ for $30 \mathrm{sec}$.
Statistical analysis. A non-parametric Mann-Whitney U test was performed using Excel 1810 software 2018 (Microsoft Corporation, Redmond, WA, USA) and one-way analysis of variance (ANOVA) followed by Tukey's post hoc test was carried out using One-way ANOVA with post-hoc Tukey HSD test calculator (http://www.astatsa.com). $\mathrm{P}<0.05$ was considered to indicate a statistically significant difference. Data are presented as the mean \pm standard deviation. All experiments were independently repeated a minimum of three times.

\section{Results}

PDE4D is a major regulator of cAMP expression levels in DLD-1 cells. Considering that previous studies demonstrated a potential role of cAMP signaling in the survival of CRC cells $(22,25)$, the present study investigated how intracellular cAMP expression levels are affected by forskolin, a chemical activator of adenylyl cyclase. Forskolin alone demonstrated an insignificant effect (Fig. 1A), which indicates that cAMP-degrading PDEs are present. It was identified that treatment with rolipram, a selective PDE4 inhibitor, in combination with forskolin, was associated with a significant increase in cAMP levels, which indicates that PDE4 is a major hydrolyzer of cAMP. Notably, treatment with rolipram alone did not increase cAMP expression levels, which may be due to low basal activities of adenylyl cyclase. Subsequently, RT-qPCR was performed, which identified that the PDE4D mRNA level was significantly increased, compared with PDE4A, 4B or 4C, which indicates that PDE4D is primarily responsible for CAMP degradation in DLD-1 CRC cells (Fig. 1B). However, other cAMP PDEs, including PDE3 and PDE7, may serve a role.

A previous study characterized the cAMP response elements (CREs) in the PDE4D promoter region using human airway smooth muscle cells (26). To investigate whether the expression of PDE4D is responsive to cAMP in DLD-1 cells, the present study exposed cells to forskolin, rolipram or a combination of forskolin and rolipram to modulate intracellular cAMP levels. The administration of single agents exhibited no significant effect on PDE4D expression; however, co-treatment with forskolin and rolipram significantly increased the expression level of PDE4D by $>3$-fold (Fig. 1C). This data is associated with cAMP concentrations upon treatment with these drugs, with forskolin/rolipram combination, but not forskolin or rolipram treatment alone, resulting in increased cAMP levels, indicating that PDE4D expression is regulated by cAMP in DLD-1 cells. The expression levels of other PDE4 isoforms, including PDE4A, 4B and 4C, were significantly reduced compared with $\mathrm{PDE} 4 \mathrm{D}$; however, their expression levels in response to forskolin, rolipram or a combination were also examined. As demonstrated in Fig. 1C, PDE4B mRNA levels significantly increased upon exposure to forskolin or forskolin/rolipram. This result agrees with a previous study, which demonstrated that CREs exist in the promoter region of PDE4B, but not PDE4A (27). To the best of our knowledge, the presence or absence of CREs in the PDE4C promoter region remains to be characterized.

Suppression of the AKT/mTOR/Myc signaling pathway by cAMP enhances oncogenic properties of DLD-1 cells. The aforementioned data demonstrated that only a combination of 
A

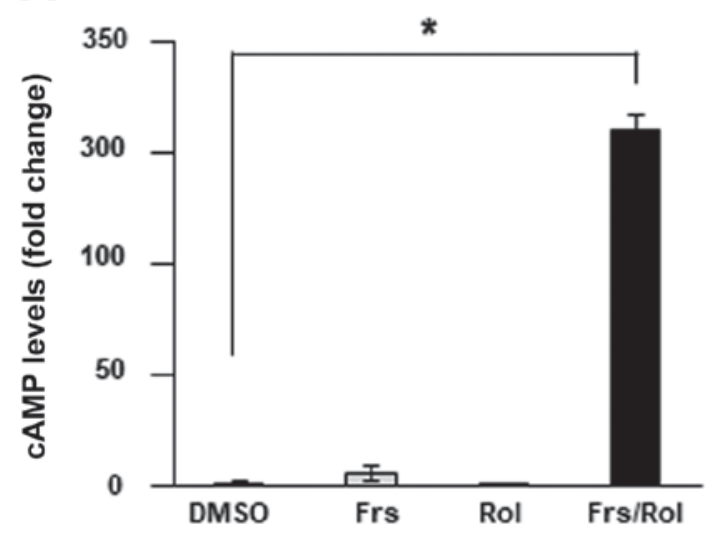

B

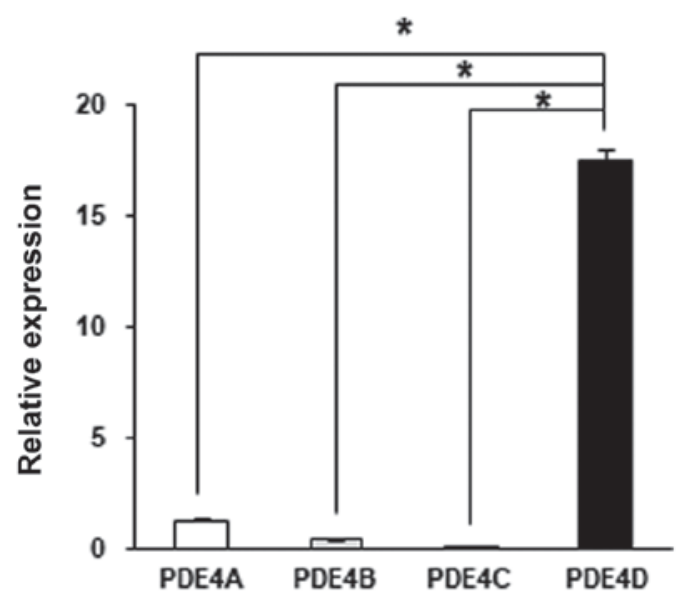

C

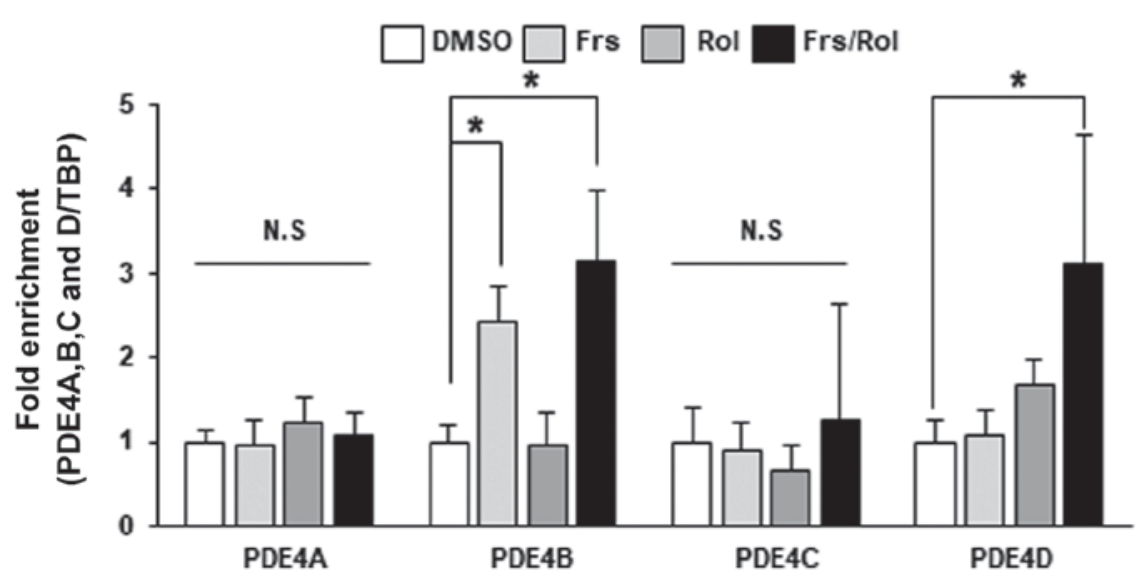

Figure 1. Intracellular cAMP levels are modulated by PDE4D in DLD-1 CRC cells. (A) Intracellular cAMP expression levels were measured in PDE4D-high DLD-1 cells following the addition of DMSO, $40 \mu \mathrm{M}$ Frs for $2 \mathrm{~h}, 40 \mu \mathrm{M}$ Rol for $6 \mathrm{~h}$, or a combination of Frs and Rol. Data are presented relative to treatment with DMSO. (B) Expression levels of PDE4A, 4B, 4C and 4D were analyzed by reverse transcription-quantitative polymerase chain reaction. (C) PDE4D mRNA levels were analyzed in DLD-1 CRC following treatment with DMSO, $40 \mu \mathrm{M}$ Frs for $2 \mathrm{~h}, 40 \mu \mathrm{M}$ Rol for $6 \mathrm{~h}$, or a combination of Frs and Rol. Data are presented as the mean \pm standard deviation. ${ }^{*} \mathrm{P}<0.05$, according to one-way analysis of variance. cAMP, cyclic adenosine 3',5'-monophosphate; PDE, phosphodiesterase; CRC, colorectal cancer; Frs, forskolin; Rol, rolipram; TBP, TATA-box binding protein; DMSO, dimethyl sulfoxide.

forskolin with rolipram significantly increased cAMP concentrations, whereas either agent alone exhibited no significant effect (Fig. 1). Subsequently, the present study examined whether the increase in cAMP levels by combinatory treatment affects the phenotype in DLD-1 cells. Clonogenic and soft agar assays demonstrated that treatment with forskolin/rolipram significantly inhibited colony formation, cell proliferation and anchorage-independent growth, which indicates that cAMP exhibits an inhibitory effect on the malignant properties of these cells (Fig. 2A-C). Roflumilast, an FDA-approved PDE4 inhibitor (28), when combined with forskolin, exhibited the same effect as rolipram (Fig. 2D-F). In summary, the data demonstrated that high cAMP concentrations can suppress the oncogenic properties of DLD-1 cells, which indicates that these cells may typically exhibit low cAMP levels.

Subsequently, the present study aimed to identify the signaling pathways that mediate the tumor-suppressive activities of cAMP. It has previously been demonstrated that the AKT/mTOR pathway and Myc, a well-characterized downstream target, regulate the survival of CRC cells (29-31). Additionally, the activities of AKT/mTOR signaling and the expression of Myc have been revealed to be regulated by cAMP in different types of cells, including human diffuse large B cell lymphoma cell lines $(19,32,33)$. The present study hypothesized that a downregulation of the AKT/mTOR/Myc axis by increased cAMP levels may inhibit the survival of DLD-1 CRC cells. To test this, DLD-1 CRC cells were treated with forskolin, rolipram or forskolin/rolipram, and the expression levels of pAKT, p4EBP1 and Myc were measured by western blot analysis. Treatment with either agent alone exhibited no significant effect on pAKT, p4EBP1 and Myc expression levels, which were markedly downregulated by simultaneous administration of forskolin and rolipram (Fig. 3A-C). These results support the aforementioned observed intracellular cAMP levels upon the addition of these agents both when used separately and in combination. Specifically, co-treatment with forskolin and rolipram resulted in a significant increase in cAMP levels while either agent alone exhibited no significant effect (Fig. 1A), which indicates that cAMP inhibits the AKT/mTOR/Myc signaling pathway, which is associated with the regulation of the malignant properties of DLD-1 CRC cells. To confirm cAMP's negative impact on this signaling pathway, western blot analysis was also performed using another PDE4 inhibitor roflumilast, which demonstrated a similar effect on 
A
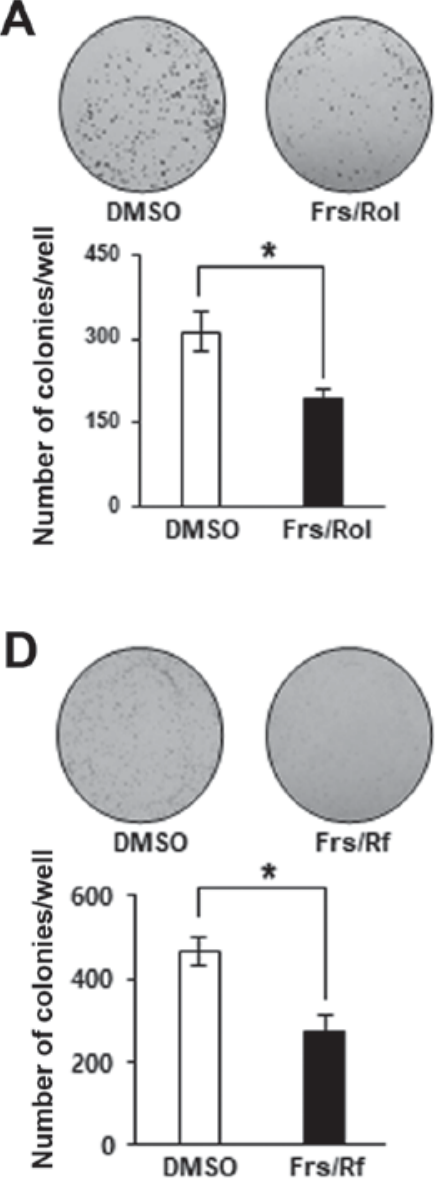

B

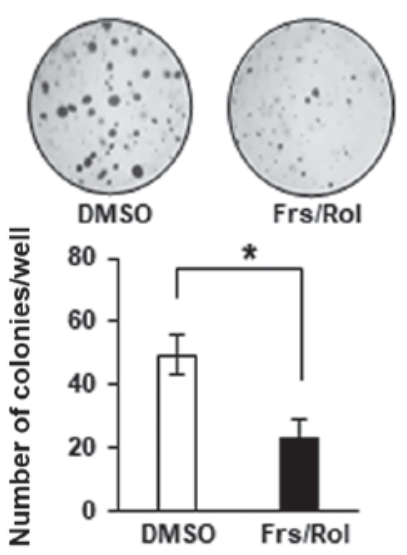

E

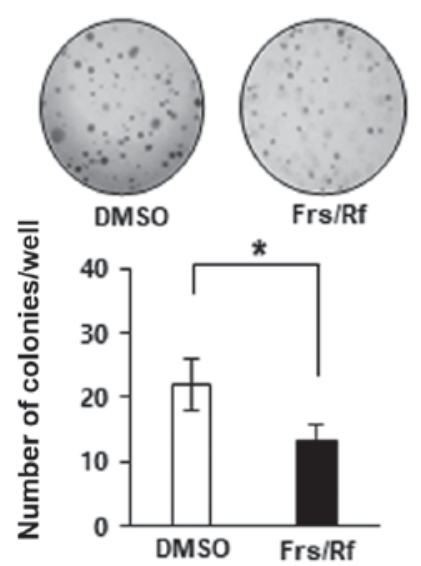

C

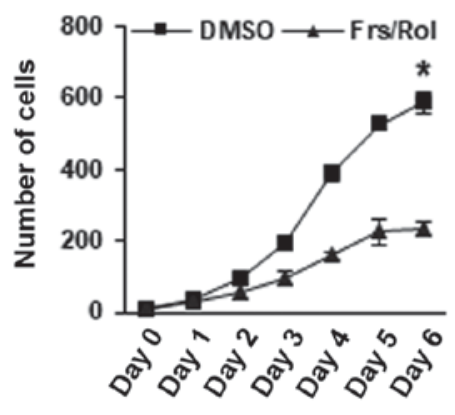

F

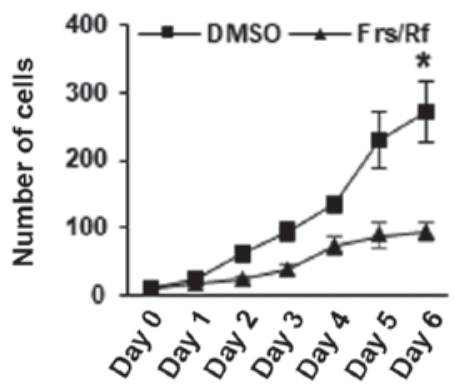

Figure 2. Cyclic adenosine 3',5'-monophosphate/phosphodiesterase 4D signals affect the oncogenic phenotypes of DLD-1 cells. (A) For colony formation assays, DLD-1 cells were seeded in 6-well plates followed by treatment with DMSO, or a combination of $20 \mu \mathrm{M}$ Frs and $40 \mu \mathrm{M}$ Rol for 7 days. The colonies were stained with $0.5 \%$ crystal violet and counted at x100 magnification. (B) For soft agar assays, DLD-1 cells were seeded in soft agar and treated with DMSO, or a combination of $20 \mu \mathrm{M}$ Frs and $40 \mu \mathrm{M}$ Rol. Following 21 days, $0.005 \%$ crystal violet was added and the colonies were counted at $\mathrm{x} 20$ magnification. (C) For cell counting, DLD-1 cells were counted at x100 magnification every day for 6 days while treating with either DMSO, or a combination of $20 \mu$ M Frs and $40 \mu \mathrm{M}$ Rol. (D) For colony formation assays, DLD-1 cells were seeded in 6-well plates followed by treatment with DMSO, or a combination of $20 \mu \mathrm{M}$ Frs and $40 \mu \mathrm{M}$ Rf for 7 days. The colonies were counted at x100 magnification following staining with $0.5 \%$ crystal violet. (E) For soft agar assays, DLD-1 cells were seeded in soft agar and treated with DMSO, or a combination of $20 \mu \mathrm{M}$ Frs and $40 \mu \mathrm{M}$ Rf. After 21 days, $0.005 \%$ crystal violet was added and the colonies were counted at x20 magnification. (F) For cell counting, DLD-1 cells were counted at x100 magnification every day for 6 days, while treated with either DMSO, or a combination of $20 \mu \mathrm{M}$ Frs and $40 \mu \mathrm{M}$ Rf. Data are presented as the mean \pm standard deviation. ${ }^{*} \mathrm{P}<0.05 \mathrm{vs}$. DMSO, according to Mann-Whitney U test. Frs, forskolin; Rol, rolipram; Rf, Roflumilast; DMSO, dimethyl sulfoxide.

pAKT, p4EBP1 and Myc expression levels, compared with rolipram (Fig. 3D). Treatment with forskolin/roflumilast decreased the expression levels of pAKT, p4EBP1 and Myc. In summary, these data indicate that the oncogenic phenotype of DLD-1 cells is predominantly suppressed by cAMP through inhibition of the AKT/mTOR/Myc pathway; however, the possibility that cAMP modulates the survival of DLD-1 cells via other signaling pathways cannot be excluded.

mTOR-Myc axis serves a critical role in regulating the malignant phenotype of DLD-1 cells. To further characterize the mediators that regulate the oncogenic properties of DLD-1 cells, the present study used rapamycin and JQ1, chemical inhibitors of mTOR and bromo- and extra-terminal domain (BET) proteins (34), respectively. Treatment with rapamycin decreased the expression levels of Myc and p4EBP1, which indicates that Myc is a downstream target of mTOR signaling (Fig. 4A). Additionally, the inhibition of the mTOR/Myc axis by rapamycin was associated with a significant decrease in the number of colonies and the number of cells in proliferation assays (Fig. 4B-D). As expected, JQ1 also decreased the expression level of Myc and demonstrated a similar effect on colony number and cell number, compared with rapamycin (Fig. 4), which indicates that mTOR and Myc are critical mediators of the antitumor effect of cAMP in DLD-1 cells.

Resveratrolreproduces the antitumor effect of PDE4 inhibitors. Based on previous studies that demonstrated that resveratrol, a type of natural phenol, inhibits PDE4 activities $(35,36)$, the present study investigated whether resveratrol could exhibit similar effects to the selective chemical inhibitors rolipram and roflumilast. Following treatment with resveratrol and/or forskolin, the intracellular levels of cAMP were measured. Combined treatment with resveratrol and forskolin significantly increased the expression level of cAMP in DLD-1 cells, compared with the control; however, the addition of either agent alone did not exhibit any significant effect (Fig. 5A). This confirms that resveratrol exhibits PDE4-inhibitor activities. 


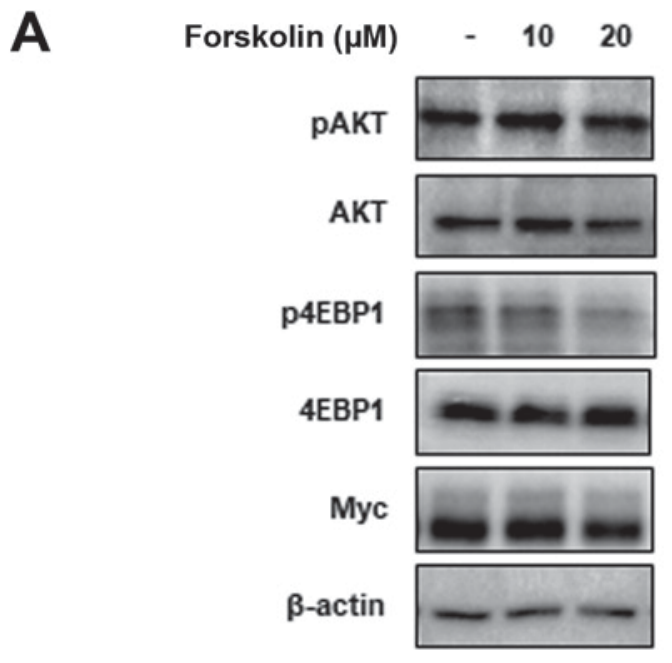

C

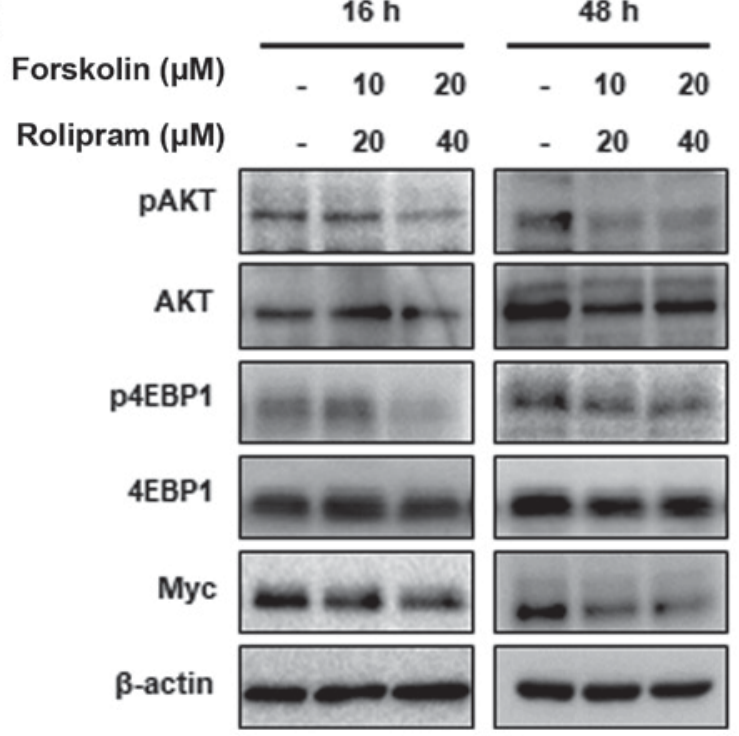

B

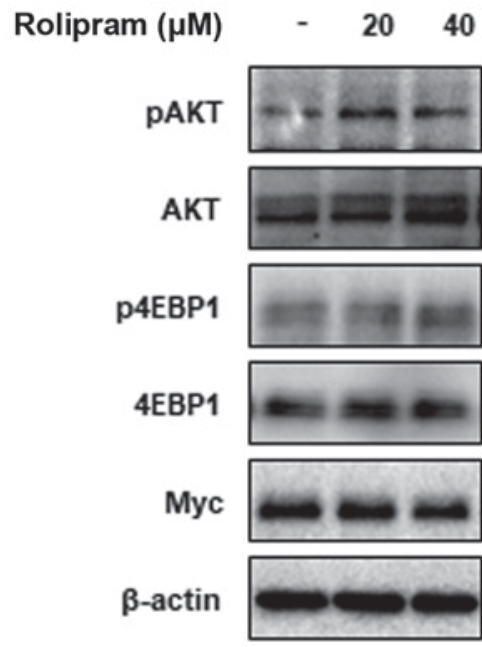

D

Forskolin $(\mu \mathrm{M})$

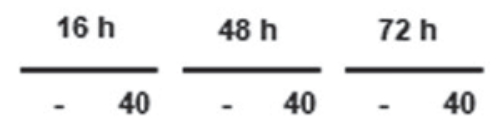

Roflumilast $(\mu \mathrm{M})$

- 40
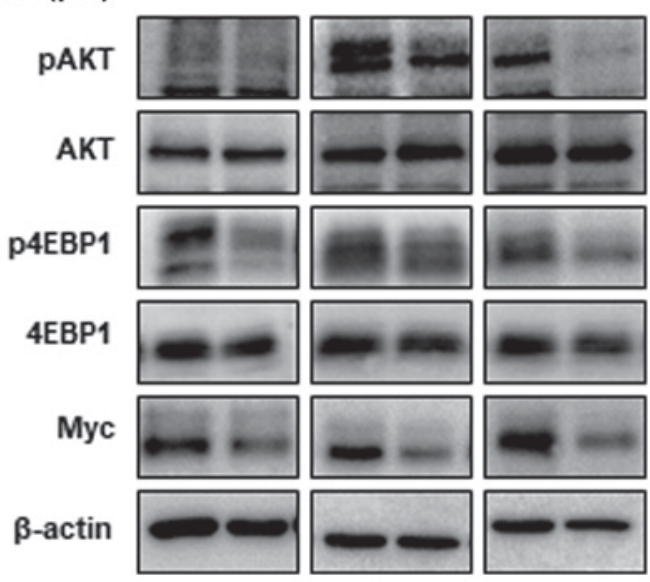

Figure 3. Cyclic adenosine 3',5'-monophosphate/PDE4D signaling regulates the activities of the AKT/mammalian target of rapamycin/Myc pathway. Western blot assays were performed to examine the phosphorylation levels of AKT and 4EBP1, and the expression of Myc following treatment with (A) forskolin $(0-20 \mu \mathrm{M}$ for $16 \mathrm{~h}),(\mathrm{B})$ rolipram $(0-40 \mu \mathrm{M}$ for $16 \mathrm{~h}),(\mathrm{C})$ a combination of forskolin and rolipram, or (D) a combination of forskolin and roflumilast in PDE4D-high DLD-1 colorectal cancer cells. Western blots for $\beta$-actin confirm equal loading. The expression levels of Myc, pAKT and p4EBP1 were markedly decreased upon combined treatment with forskolin and rolipram/roflumilast, while the addition of single agents exhibited a minimal effect. PDE4D, phosphodiesterase 4D; p, phosphorylated; 4EBP1, 4E-binding protein 1.

Consistent with the antitumor effect of cAMP, an increase in cAMP levels following the administration of resveratrol and forskolin was associated with decreased levels of p4EBP1 and Myc (Fig. 5B). This in turn was associated with suppression of cell proliferation and colony formation in DLD-1 cells (Fig. 5C and D). These data indicate that resveratrol may be an effective therapeutic agent for CRC with high PDE4 activity.

\section{Discussion}

To the best of our knowledge, for the first time the present study demonstrated that aberrant PDE4D expression may contribute to the malignant phenotype of DLD-1 CRC cells. Notably, the mTOR-Myc axis was identified to serve a critical role in mediating the effect of PDE4D on the oncogenic properties of these cells. Increased expression levels of cAMP following treatment with PDE4 inhibitors suppressed mTOR signaling and Myc expression. Additionally, the mTOR inhibitor, rapamycin, and BET bromodomain inhibitor, JQ1, exhibited similar tumor suppressive effects, compared with PDE4 inhibitors. cAMP, an important second messenger, regulates diverse processes, including cell survival and death, in a cell type- and context-dependent manner. It has been demonstrated that numerous types of cancer cells prefer low levels of cAMP for their growth and survival, which can be reversed using inhibitors of cAMP-specific PDEs $(25,37,38)$. The present study is in agreement with these previous results, which demonstrates that cancer cells rely on low cAMP levels.

Previous studies have reported that regulation of mTOR phosphorylation/activity depends on the cell type. For example, an increase of cAMP levels led to inhibition of mTOR activity in 3T3-L1 adipocytes and B lymphoma cells $(19,39)$, as has been observed in CRC cells. However, mTOR activity was 
A

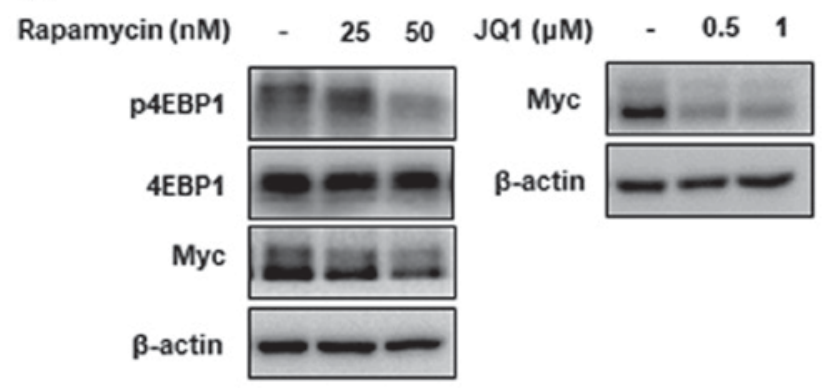

C
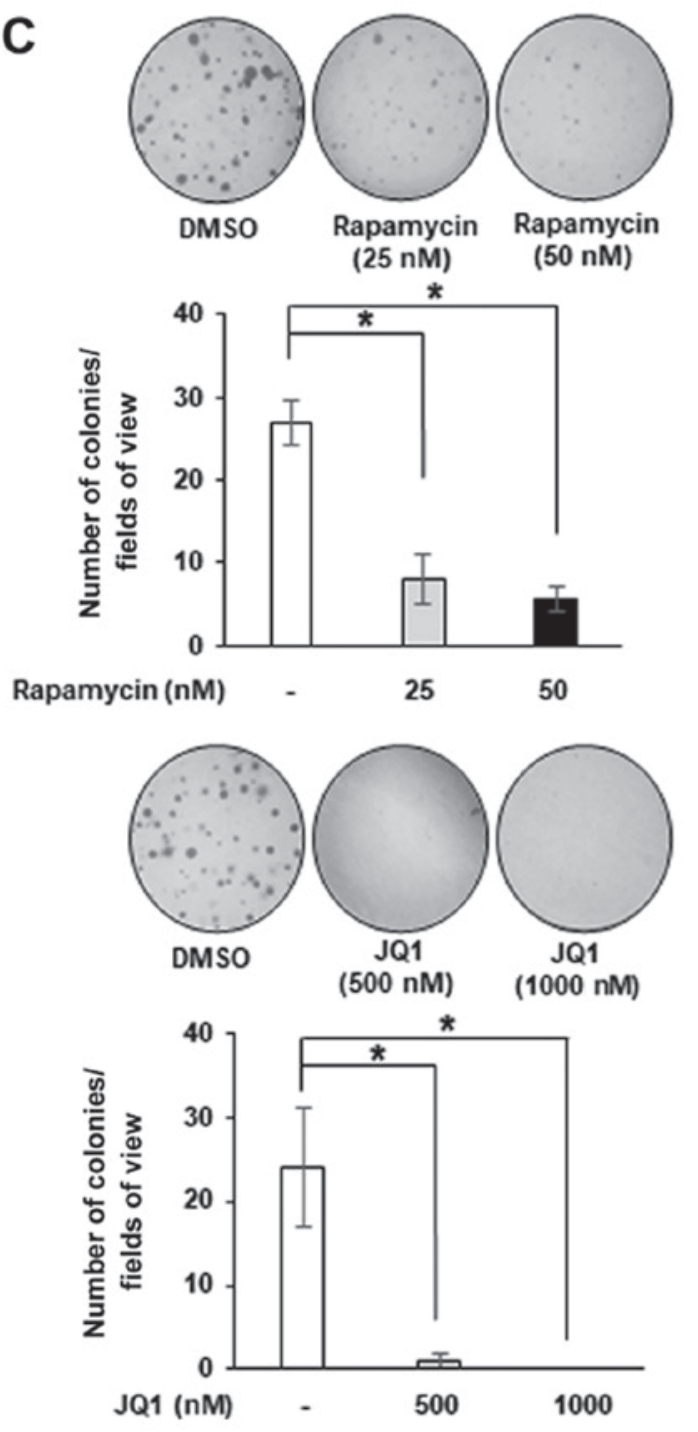

B
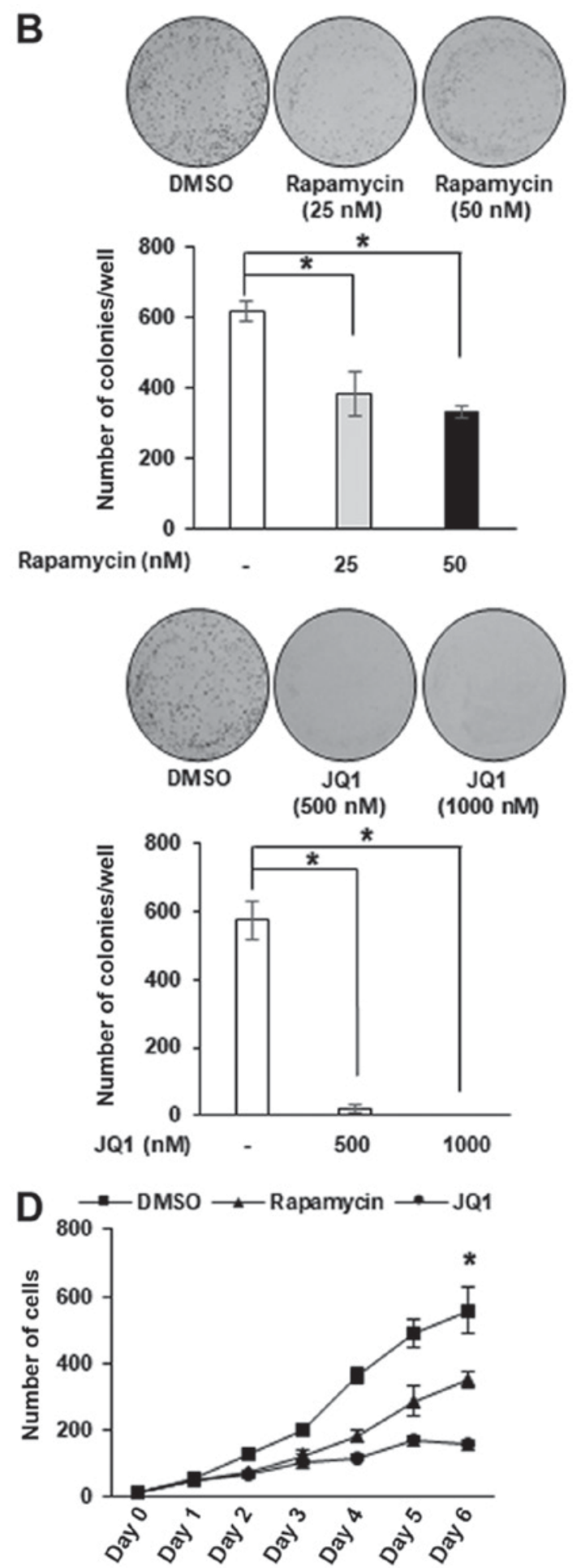

Figure 4. JQ1 and rapamycin duplicate the inhibitory effect of cyclic adenosine 3',5'-monophosphate in DLD-1 cells. (A) DLD-1 cells were treated with rapamycin or JQ1 for $24 \mathrm{~h}$ to analyze the expression levels of p4EBP1 and Myc by western blot analysis. (B) Colony formation assay and (C) soft agar assay were performed following treatment with DMSO, rapamycin $(0-50 \mathrm{nM})$ or JQ1 $(0-1,000 \mathrm{nM})$ and counted with the naked eye and at $\mathrm{x} 20 \mathrm{magnification}$. ${ }^{*} \mathrm{P}<0.05$, according to one-way analysis of variance. (D) Cell counting was performed following treatment with DMSO, rapamycin (0-50 nM) or JQ1 (0-1,000 nM). Statistical significance for each drug-treated group was compared with DMSO treatment. * $\mathrm{P}<0.05$, according to one-way analysis of variance. Data are presented as the mean \pm standard deviation. p, phosphorylated; 4EBP1, 4E-binding protein 1; DMSO, dimethyl sulfoxide.

enhanced by cAMP in PCCL3 rat thyroid cells, which was demonstrated by an increase in ribosomal protein S6 kinase $\beta 1$ and 4EBP1 phosphorylation (40). It would be beneficial to examine what determines the cell type-dependent regulation of mTOR activity by cAMP. The present results, which revealed a downregulation of mTOR and Myc by roflumilast, may exhibit clinical implications. Roflumilast was FDA-approved for the treatment of chronic obstructive pulmonary disease in 2011 and a clinical trial has successfully been performed to test its efficacy against aggressive B-cell lymphoma (41). The present study indicates that this drug may be repurposed to suppress Myc and CRC development in a clinical setting. 

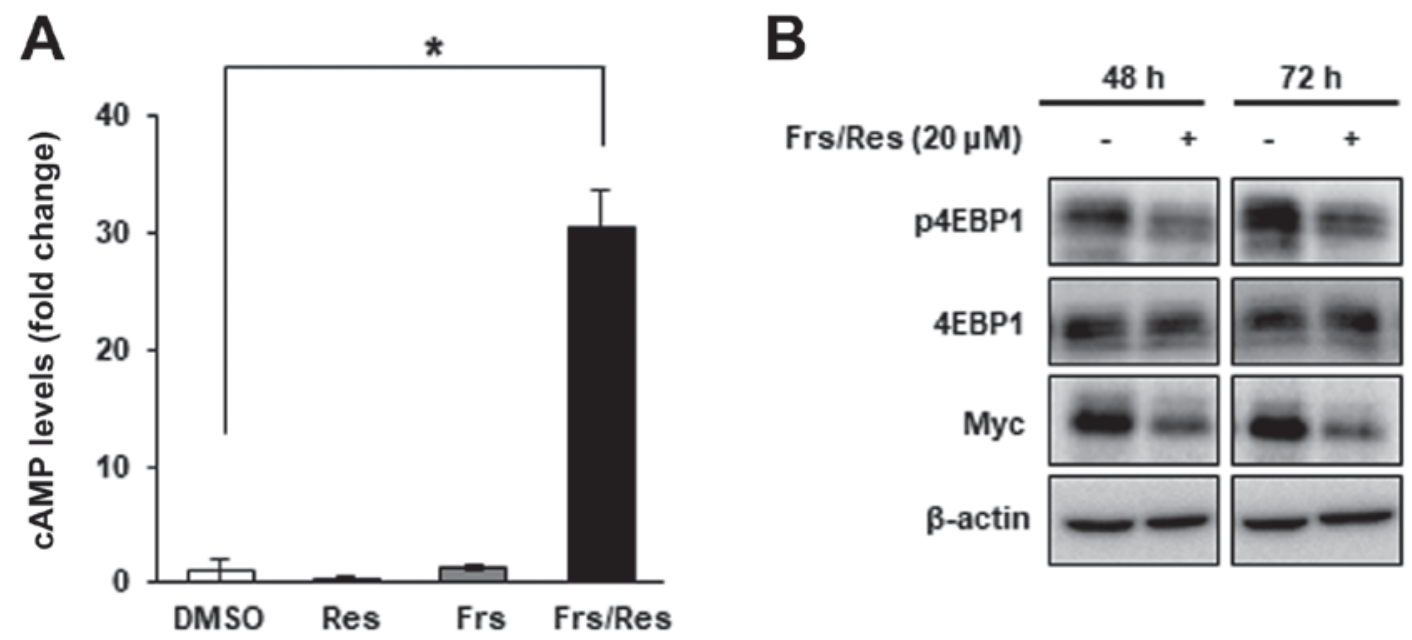
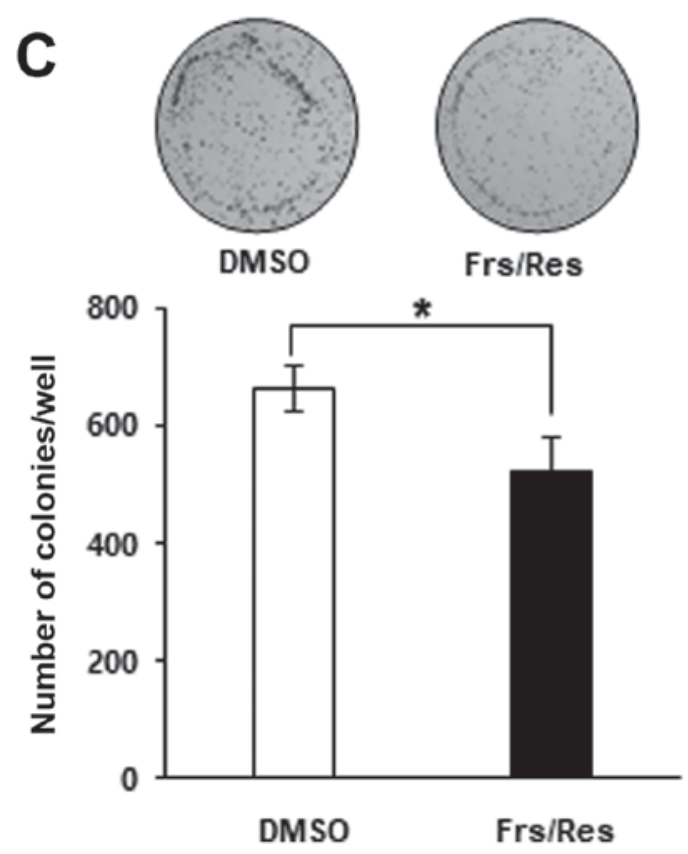

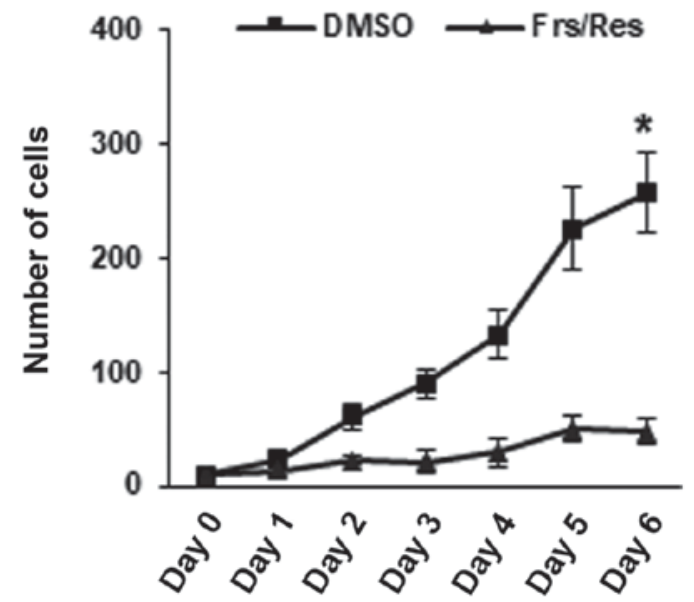

Figure 5. Res reproduces the effect of rolipram and roflumilast in DLD-1 cells. (A) DLD-1 colorectal cancer cells were treated with DMSO, $20 \mu \mathrm{M}$ Res for $14 \mathrm{~h}, 20 \mu \mathrm{M}$ Frs for $2 \mathrm{~h}$ or a combination of Frs and Res, and the intracellular cAMP levels were measured. Data are presented as fold changes relative to the cAMP levels in cells treated with DMSO. Co-treatment of Res and Frs significantly increased cAMP levels while treatment of either agent alone exhibited no significant effect ${ }^{*} \mathrm{P}<0.05$, according to one-way analysis of variance. (B) Expression levels of Myc and p4EBP1 were analyzed following treatment with either DMSO, or a combination of $20 \mu \mathrm{M}$ Frs and $20 \mu \mathrm{M}$ Res for 48 or $72 \mathrm{~h}$. (C) Colony formation was analyzed following treatment with either DMSO, or a combination of $20 \mu \mathrm{M}$ Frs and $20 \mu \mathrm{M}$ Res and counted with the naked eye. ${ }^{*} \mathrm{P}<0.05$, according to Mann-Whitney $\mathrm{U}$ test. (D) Cell proliferation was analyzed following treatment with either DMSO, or a combination of $20 \mu \mathrm{M}$ Frs and $20 \mu \mathrm{M}$ Res. "P $<0.05$ vs. Frs/Res, according to Mann-Whitney U test. Data are presented as the mean \pm standard deviation. Res, resveratrol; Frs, forskolin; cAMP, cyclic adenosine 3',5'-monophosphate; p, phosphorylated; 4EBP1, 4E-binding protein 1; DMSO, dimethyl sulfoxide.

PDE4D has been identified to affect tumor progression in a number of ways in different types of cancer. For example, PDE4D interacts with focal adhesion kinase via the receptor for activated $\mathrm{C}$ kinase 1 scaffold protein to regulate cell invasion in BRAF-mutated melanoma (42). In medulloblastoma, inhibition of PDE4D downregulates Hedgehog (Hh) signaling to suppress tumor growth, indicating that PDE4D can be a therapeutic target in tumors with high $\mathrm{Hh}$ activities (43).

Myc has been demonstrated to control several important aspects of cancer cell biology, including promoting cell proliferation, inducing resistance to chemotherapeutic drug-induced apoptosis and it is associated with invasion/metastasis and metabolic reprogramming (44). As demonstrated by cell counting and soft agar assays, increased cAMP due to PDE4D inhibition suppressed cancer cell proliferation and invasion in vitro. Testing the efficacy of PDE4D inhibitors in vivo using animal models may provide improved insight into the role of PDE4D in the pathogenesis of colon cancer. GEBR-7b and GEBR-32a are two newly developed PDE4D inhibitors $(45,46)$. These compounds have demonstrated memory-enhancing activities in animal models and may be used in the therapies of neurodegenerative disorders, including Alzheimer's disease (46). 
Additionally, GEBR-7b has been used to prevent tamoxifen resistance in ER-positive breast cancer in vivo (47); however, the tumor-suppressive effect of these inhibitors has not been investigated in colon cancer, which requires further studies.

It has been demonstrated that PDE4D is aberrantly expressed in patients with prostate cancer and tamoxifen-resistant breast cancer cells $(47,48)$. Although a more systematic approach is required to reach any substantial conclusion, the RT-qPCR data indicated that DLD-1 cells highly express PDE4D. This indicates that $\mathrm{CRC}$ cells and patients with CRC may also exhibit abnormal PDE4D levels, which may potentially affect the pathogenesis of the disease. The mechanisms underlying PDE4D overexpression in CRC remain to be elucidated. However, recent data indicated that downregulation of miR-139-5p may serve a role in elevated levels of PDE4D. Firstly miRNA-139-5p induced by the 553 tumor suppressor has been demonstrated to target PDE4D in cancer cells (23). Additionally, the expression of miR-139-5p was markedly reduced in CRC tissues, compared with adjacent non-cancerous tissues (49). Lastly, the present study revealed that the expression levels of miR-139-5p and PDE4D were inversely correlated in CRC tissue samples. Further studies may improve the understanding regarding the mechanisms underlying PDE4D overexpression in CRC and other types of cancer.

Protein kinase A (PKA) and exchange protein activated by cAMP (EPAC) are the main effectors of cAMP (50); however, it is unclear whether the anti-proliferative effect of cAMP in DLD-1 cells is dependent on PKA and/or EPAC. Notably, the cytotoxic effects of cAMP in normal and malignant $\mathrm{B}$ cells are independent of PKA and EPAC (21). Additionally, activation of cAMP signaling by loss of PDE4D mediates resistance to the chemotherapeutic drug Triapine via EPAC in the SW480 human colon adenocarcinoma cell line (51). These data indicate that cAMP signaling is performed in a cell type- and context-dependent manner. It would be beneficial to examine downstream target molecules of cAMP that mediate its tumor-suppressive effect in DLD-1 cells.

Resveratrol is a natural polyphenolic compound present in red wine and other food products. It is an antioxidant with potential antitumor and anti-aging properties (35). In a murine aging model, treatment with resveratrol reversed aging-associated metabolic abnormalities, including diet-induced obesity and impaired glucose tolerance, which has been identified to be reproduced by a PDE4 inhibitor rolipram (35). The present study demonstrated that resveratrol can suppress the malignant phenotype of DLD-1 cells with high expression of PDE4D. Previous data, together with the present results, clearly indicate that PDE4D is a promising target for the treatment of patients with cancer with aberrant expression of PDE4D.

\section{Acknowledgements}

Not applicable.

\section{Funding}

This work was supported by Basic Science Research Program through the National Research Foundation of Korea funded by the Ministry of Science, ICT and Future Planning
(NRF-2013R1A1A2008838 and NRF-2016R1A2B4011758) to SWK.

\section{Availability of data and materials}

All data generated or analyzed during this study are included in this published article.

\section{Authors' contributions}

DUK designed and performed the experiments, analyzed the data and wrote the manuscript. JN performed experiments and analyzed the data. MDC performed statistical analysis of all data and assisted with revision of the manuscript. SWK designed and supervised the study, analyzed the data and wrote the manuscript. All the authors read and approved the final manuscript.

\section{Ethics approval and consent to participate}

Not applicable.

\section{Patient consent for publication}

Not applicable.

\section{Competing interests}

The authors declare that they have no competing interests.

\section{References}

1. Arnold M, Sierra MS, Laversanne M, Soerjomataram I, Jemal A and Bray F: Global patterns and trends in colorectal cancer incidence and mortality. Gut 66: 683-691, 2017.

2. Siegel RL, Miller KD and Jemal A: Colorectal cancer mortality rates in adults aged 20 to 54 years in the united states, 1970-2014. JAMA 318: 572-574, 2017.

3. Worthley DL and Leggett BA: Colorectal cancer: Molecular features and clinical opportunities. Clin Biochem Rev 31: 31-38, 2010.

4. Hinoue T, Weisenberger DJ, Pan F, Campan M, Kim M, Young J, Whitehall VL, Leggett BA and Laird PW: Analysis of the association between CIMP and BRAF in colorectal cancer by DNA methylation profiling. PLoS One 4: e8357, 2009.

5. Mundade R, Imperiale TF, Prabhu L, Loehrer PJ and Lu T: Genetic pathways, prevention, and treatment of sporadic colorectal cancer. Oncoscience 1: 400-406, 2014.

6. Walther A, Johnstone E, Swanton C, Midgley R, Tomlinson I and Kerr D: Genetic prognostic and predictive markers in colorectal cancer. Nat Rev Cancer 9: 489-499, 2009.

7. Fodde R: The APC gene in colorectal cancer. Eur J Cancer 38: 867-871, 2002.

8. Schneikert J and Behrens J: The canonical Wnt signalling pathway and its APC partner in colon cancer development. Gut 56: 417-425, 2007.

9. Easwaran V, Lee SH, Inge L, Guo L, Goldbeck C, Garrett E, Wiesmann M, Garcia PD, Fuller JH, Chan V, et al: Beta-Catenin regulates vascular endothelial growth factor expression in colon cancer. Cancer Res 63: 3145-3153, 2003.

10. Herbst A, Jurinovic V, Krebs S, Thieme SE, Blum H, Göke B and Kolligs FT: Comprehensive analysis of $\beta$-catenin target genes in colorectal carcinoma cell lines with deregulated Wnt/ $\beta$-catenin signaling. BMC Genomics 15: 74, 2014.

11. Morin PJ: Beta-catenin signaling and cancer. Bioessays 21: 1021-1030, 1999.

12. Sansom OJ, Meniel VS, Muncan V, Phesse TJ, Wilkins JA, Reed KR, Vass JK, Athineos D and Clevers H: Myc deletion rescues Apc deficiency in the small intestine. Nature 446: 676-679, 2007. 
13. Sewastianik T, Prochorec-Sobieszek M, Chapuy B and Juszczyński P: MYC deregulation in lymphoid tumors: Molecular mechanisms, clinical consequences and therapeutic implications. Biochim Biophys Acta 1846: 457-467, 2014.

14. Rall TW and Sutherland EW: Formation of a cyclic adenine ribonucleotide by tissue particles. J Biol Chem 232: 1065-1076, 1958

15. Sutherland EW and Rall TW: Fractionation and characterization of a cyclic adenine ribonucleotide formed by tissue particles. J Biol Chem 232: 1077-1091, 1958.

16. Daniel PB, Walker WH and Habener JF: Cyclic AMP signaling and gene regulation. Annu Rev Nutr 18: 353-383, 1998.

17. Fimia GM and Sassone-Corsi P: Cyclic AMP signalling. J Cell Sci 114: 1971-1972, 2001.

18. Sassone-Corsi P: The cyclic AMP pathway. Cold Spring Harb Perspect Biol 4: pii: a011148, 2012.

19. Kim SW, Rai D and Aguiar RC: Gene set enrichment analysis unveils the mechanism for the phosphodiesterase 4B control of glucocorticoid response in B-cell lymphoma. Clin Cancer Res 17: 6723-6732, 2011.

20. Livak KJ and Schmittgen TD: Analysis of relative gene expression data using real-time quantitative PCR and the 2(-Delta Delta C(T)) method. Methods 25: 402-408, 2001.

21. Smith PG, Wang F, Wilkinson KN, Savage KJ, Klein U, Neuberg DS, Bollag G, Shipp MA and Aguiar RC: The phosphodiesterase PDE4B limits cAMP-associated PI3K/AKT-dependent apoptosis in diffuse large B-cell lymphoma. Blood 105: 308-316, 2005 .

22. Tsunoda T, Ota T, Fujimoto T, Doi K, Tanaka Y, Yoshida Y, Ogawa M, Matsuzaki H, Hamabashiri M, Tyson DR, et al Inhibition of phosphodiesterase-4 (PDE4) activity triggers luminal apoptosis and AKT dephosphorylation in a 3-D colonic-crypt model. Mol Cancer 11: 46, 2012.

23. Cao B, Wang K, Liao JM, Zhou X, Liao P, Zeng SX, He M, Chen $\mathrm{L}, \mathrm{He} \mathrm{Y}, \mathrm{Li} \mathrm{W}$ and $\mathrm{Lu} \mathrm{H}$ : Inactivation of oncogenic cAMP-specific phosphodiesterase 4D by miR-139-5p in response to p53 activation. Elife 5: pii: e15978, 2016.

24. Lakics V, Karran EH and Boess FG: Quantitative comparison of phosphodiesterase mRNA distribution in human brain and peripheral tissues. Neuropharmacology 59: 367-374, 2010.

25. McEwan DG, Brunton VG, Baillie GS, Leslie NR, Houslay MD and Frame MC: Chemoresistant KM12C colon cancer cells are addicted to low cyclic AMP levels in a phosphodiesterase 4-regulated compartment via effects on phosphoinositide 3-kinase. Cancer Res 67: 5248-5257, 2007.

26. Le Jeune IR, Shepherd M, Van Heeke G, Houslay MD and Hall IP: Cyclic AMP-dependent transcriptional up-regulation of phosphodiesterase 4D5 in human airway smooth muscle cells. Identification and characterization of a novel PDE4D5 promoter. J Biol Chem 277: 35980-35989, 2002.

27. Takahashi M, Terwilliger R, Lane C, Mezes PS, Conti M and Duman RS: Chronic antidepressant administration increases the expression of cAMP-specific phosphodiesterase 4A and 4B isoforms. J Neurosci 19: 610-618, 1999.

28. Lipworth BJ: Phosphodiesterase-4 inhibitors for asthma and chronic obstructive pulmonary disease. Lancet 365: 167-175, 2005

29. Francipane MG and Lagasse E: mTOR pathway in colorectal cancer: An update. Oncotarget 5: 49-66, 2014.

30. Pandurangan AK: Potential targets for prevention of colorectal cancer: A focus on PI3K/Akt/mTOR and Wnt pathways. Asian Pac J Cancer Prev 14: 2201-2205, 2013

31. Sipos F, Firneisz G and Műzes G: Therapeutic aspects of c-MYC signaling in inflammatory and cancerous colonic diseases. World J Gastroenterol 22: 7938-7950, 2016.

32. Pirson I, Coulonval K, Lamy F and Dumont JE: c-Myc expression is controlled by the mitogenic cAMP-cascade in thyrocytes. J Cell Physiol 168: 59-70, 1996.

33. Williamson EA, Burgess GS, Eder P, Litz-Jackson S and Boswell HS: Cyclic AMP negatively controls c-myc transcription and G1 cell cycle progression in p210 BCR-ABL transformed cells: Inhibitory activity exerted through cyclin D1 and cdk4. Leukemia 11: 73-85, 1997.

34. Di Costanzo A, Del Gaudio N, Migliaccio A and Altucci L: Epigenetic drugs against cancer: An evolving landscape. Arch Toxicol 88: 651-668, 2014.
35. Park SJ, Ahmad F, Philp A, Baar K, Williams T, Luo H, Ke H, Rehmann H, Taussig R, Brown AL, et al: Resveratrol ameliorates aging-related metabolic phenotypes by inhibiting cAMP phosphodiesterases. Cell 148: 421-433, 2012.

36. Tsunoda T, Ishikura S, Doi K, Matsuzaki H, Iwaihara $Y$ and Shirasawa S: Resveratrol induces luminal apoptosis of human colorectal cancer HCT116 cells in three-dimensional culture. Anticancer Res 34: 4551-4555, 2014

37. Goldhoff P, Warrington NM, Limbrick DD Jr, Hope A, Woerner BM, Jackson E, Perry A, Piwnica-Worms D and Rubin JB: Targeted inhibition of cyclic AMP phosphodiesterase-4 promotes brain tumor regression. Clin Cancer Res 14: 7717-7725, 2008

38. Ogawa R, Streiff MB, Bugayenko A and Kato GJ: Inhibition of PDE4 phosphodiesterase activity induces growth suppression, apoptosis, glucocorticoid sensitivity, p53, and p21(WAF1/CIP1) proteins in human acute lymphoblastic leukemia cells. Blood 99: 3390-3397, 2002

39. Scott PH and Lawrence JC Jr: Attenuation of mammalian target of rapamycin activity by increased cAMP in 3T3-L1 adipocytes. J Biol Chem 273: 34496-34501, 1998.

40. Blancquaert S, Wang L, Paternot S, Coulonval K, Dumont JE, Harris TE and Roger PP: cAMP-dependent activation of mammalian target of rapamycin (mTOR) in thyroid cells. Implication in mitogenesis and activation of CDK4. Mol Endocrinol 24: $1453-1468,2010$

41. Kelly K, Mejia A, Suhasini AN, Lin AP, Kuhn J, Karnad AB, Weitman S and Aguiar RC: Safety and pharmacodynamics of the PDE4 inhibitor roflumilast in advanced B-cell malignancies. Clin Cancer Res 23: 1186-1192, 2017.

42. Delyon J, Servy A, Laugier F, André J, Ortonne N, Battistella M, Mourah S, Bensussan A, Lebbé C and Dumaz N: PDE4D promotes FAK-mediated cell invasion in BRAF-mutated melanoma. Oncogene 36: 3252-3262, 2017.

43. Ge X, Milenkovic L, Suyama K, Hartl T, Purzner T, Winans A, Meyer T and Scott MP: Phosphodiesterase 4D acts downstream of Neuropilin to control Hedgehog signal transduction and the growth of medulloblastoma. Elife 4: e07068, 2015.

44. Hanahan D and Weinberg RA: Hallmarks of cancer: The next generation. Cell 144: 646-674, 2011

45. Bruno O, Fedele E, Prickaerts J, Parker LA, Canepa E, Brullo C, Cavallero A, Gardella E, Balbi A, Domenicotti C, et al: GEBR-7b, a novel PDE4D selective inhibitor that improves memory in rodents at non-emetic doses. Br J Pharmacol 164: 2054-2063, 2011.

46. Ricciarelli R, Brullo C, Prickaerts J, Arancio O, Villa C, Rebosio C, Calcagno E, Balbi M, van Hagen BT, Argyrousi EK, et al: Memory-enhancing effects of GEBR-32a, a new PDE4D inhibitor holding promise for the treatment of Alzheimer's disease. Sci Rep 7: 46320, 2017.

47. Mishra RR, Belder N, Ansari SA, Kayhan M, Bal H, Raza U, Ersan PG, Tokat ÜM, Eyüpoğlu E, Saatci Ö, et al: Reactivation of cAMP pathway by PDE4D inhibition represents a novel druggable axis for overcoming tamoxifen resistance in ER-positive breast cancer. Clin Cancer Res 24: 1987-2001, 2018

48. Böttcher R, Dulla K, van Strijp D, Dits N, Verhoef EI, Baillie GS, van Leenders GJ, Houslay MD, Jenster G and Hoffmann R: Human PDE4D isoform composition is deregulated in primary prostate cancer and indicative for disease progression and development of distant metastases. Oncotarget 7: 70669-70684, 2016.

49. Li Q, Liang X, Wang Y, Meng X, Xu Y, Cai S, Wang Z, Liu J and Cai G: miR-139-5p inhibits the epithelial-mesenchymal transition and enhances the chemotherapeutic sensitivity of colorectal cancer cells by downregulating BCL2. Sci Rep 6: 27157, 2016.

50. Garcia-Morales V, Luaces-Regueira $\mathrm{M}$ and Campos-Toimil $\mathrm{M}$ : The cAMP effectors PKA and Epac activate endothelial NO synthase through PI3K/Akt pathway in human endothelial cells. Biochem Pharmacol 145: 94-101, 2017.

51. Miklos W, Heffeter P, Pirker C, Hager S, Kowol CR, van Schoonhoven S, Stojanovic M, Keppler BK and Berger W: Loss of phosphodiesterase 4D mediates acquired triapine resistance via Epac-Rap1-Integrin signaling. Oncotarget 7: 84556-84574, 2016. 